\title{
Reprocessing of X-rays in AGN
}

\section{Plane parallel geometry - test of pressure equilibrium}

\author{
A.-M. Dumont ${ }^{1}$, B. Czerny $^{2}$, S. Collin ${ }^{1}$, and P. T. Życki ${ }^{2}$ \\ 1 Observatoire de Paris-Meudon, DAEC, Meudon, France \\ 2 Copernicus Astronomical Center, Bartycka 18, 00-716 Warsaw, Poland
}

Received 23 January 2002 / Accepted 5 March 2002

\begin{abstract}
We present a model of the vertical stratification and the spectra of an irradiated medium under the assumption of constant pressure. Such a solution has properties intermediate between constant density models and hydrostatic equilibrium models, and it may represent a flattened configuration of gas clumps accreting onto the central black hole. Such a medium develops a hot skin, thicker than hydrostatic models, but thinner than constant density models, under comparable irradiation. The range of theoretical values of the $\alpha_{\mathrm{ox}}$ index is comparable to those from hydrostatic models and both are close to the observed values for Seyfert galaxies but lower than in quasars. The amount of X-ray Compton reflection is consistent with the observed range. The characteristic property of the model is a frequently multicomponent iron $\mathrm{K}_{\alpha}$ line.
\end{abstract}

Key words. radiative transfer - accretion disks - galaxies: active - galaxies: Seyfert - X-rays: stars X-rays: galaxies

\section{Introduction}

The intense production of hard X-ray emission is a basic property of all active galactic nuclei (AGN), although the fraction of this emission in comparison to the total bolometric luminosity is different in various types of AGN. A fraction of this radiation illuminates the relatively cold material present there and provides a reflection component apparent in X-ray spectra (Pounds et al. 1990; for a review see Mushotzky et al. 1993). The component consists of an iron $\mathrm{K}_{\alpha}$ line, usually at $6.4 \mathrm{keV}$ appropriate for only weakly ionized iron, an accompanying edge, and a Compton "hump" peaking at about $30 \mathrm{keV}$.

Above $1 \mathrm{keV}$ the spectral index is equal to 0.7 on average, and in a majority of objects the continuum below $1 \mathrm{keV}$ displays an excess when compared to the extrapolation of the continuum in the $1-10 \mathrm{keV}$ range (the so-called "soft X-ray excess"). Recent observations performed with Chandra show that the strength of the excess has probably been overestimated in the past. This soft X-ray excess may, or may not, be related to the X-ray reprocessing (e.g. Czerny \& Życki 1994; Leighly 1999).

Above $1 \mathrm{keV}$ the spectrum can be decomposed into an underlying power law (with a typical spectral index $\Gamma \sim 1.9$ ) and a reflection component. The strength of the reflection component correlates strongly with the slope of

Send offprint requests to: A.-M. Dumont, e-mail: bcz@camk.edu.pl the power law, and this effect is universal for all accreting black holes, i.e. AGN and galactic black holes (Ueda et al. 1994; Magdziarz et al. 1998; Done \& Życki 1999; Zdziarski et al. 1999; Gilfanov et al. 1999; Revnivtsev et al. 2001). Frequency-resolved spectroscopy puts additional constraints on the formation of the reflection component (Gilfanov et al. 2000; Revnivtsev et al. 1999; Życki 2002) but this powerful technique was not applied so far to AGN observations.

The iron $\mathrm{K}_{\alpha}$ line displays an asymmetrical profile with an extended red wing and a much smaller blue wing (Nandra et al. 1997). The presence of the broad red wing in the Seyfert composite spectrum has been recently questioned (Lubiński \& Zdziarski 2001). However, in the two best studied objects, MCG-6-30-15 and NGC 3516, the line wing extends down to $4 \mathrm{keV}$ (see Tanaka et al. 1995; Iwasawa et al. 1996; Lubiński \& Zdziarski 2001 for MCG6-30-15, and Nandra et al. 1999 for NGC 3516). Recent high resolution Chandra data show a neutral very narrow iron line in most objects (e.g. Yaqoob et al. 2001a; Sambruna et al. 2001; Kaspi et al. 2001). These results most probably mean that the line is multicomponent, as actually shown by Yaqoob et al. (2001b). The equivalent width of the line seems also to correlate with the spectral slope of the power law (Lubiński \& Zdziarski 2001).

The iron line and the reflection hump have been now extensively used for the diagnosis of the geometry of the accretion flow pattern close to the black hole (e.g. 
Fabian et al. 2000; Yu \& Lu 2000; Ruszkowski et al. 2000; Abrassart \& Dumont 1998, 2000; Karas et al. 2000; Życki \& Różańska 2001, and references therein).

Recent intensive monitoring campaigns brought, however, more confusion than enlightening to the emerging picture. According to predictions based on a simple analysis, a variable X-ray emission should give in response a significant variability in the optical/UV band due to the absorption of a fraction of incident X-rays. Also both the reflected X-ray continuum and the iron line should vary in concert, though with a small delay (e.g. Rokaki et al. 1993; Clavel et al. 1992; Berkley et al. 2000; Kazanas \& Nayakshin 2001). Recent results of careful monitoring, however, showed that the level of the optical/UV response is surprisingly different in various sources, the correlation between the optical/UV and X-rays is far from perfect or is actually absent, the iron line total flux responds only weakly or not at all to variable X-rays, and the level of reflection in continuum is not positively correlated with the strength of the line.

For instance, NGC 7469 shows variations of the UV and X-ray fluxes with similar large amplitudes and a time delay of the order of 2 days, the UV leading the X-rays in the flux peaks, but the X-ray flux displays in addition short term variations not seen in UV (Nandra et al. 1998). The UV flux correlates with $\Gamma$ (Nandra et al. 2000). For NGC 3516 there is no clear correlation between UV and X (Edelson et al. 2000). For NGC 4051 no optical variations were observed when strong $\mathrm{X}$-ray variations were seen (Done et al. 1990). The UV flux variations drive the optical flux variations with a time delay $\leq 0.2 \mathrm{~d}$ for NGC 4151 (Edelson et al. 1996), $\leq 0.15$ d for NGC 3516 (Edelson et al. 2000), and for NGC 7469 a delay of the order a day which increases with the wavelength (Wanders et al. 1997; Collier et al. 1998). Soft X-ray variations are generally larger than hard X-ray ones, but this is not always the case (Nandra et al. 1997). For NGC 3516, there were strongly correlated variations with no measurable lag ( $\leq 0.15$ d, Edelson et al. 2000).

The centroid energy, the intensity, and the equivalent width of the $\mathrm{Fe} \mathrm{K}_{\alpha}$ line, are variable on short time scales (down to ksec). The correlations between the continuum and the $\mathrm{Fe} \mathrm{K}_{\alpha}$ line have been intensively looked for in a few objects with ASCA and RXTE, and now with Chandra, revealing a complex behaviour of the line versus the underlying continuum. Iwasawa et al. (1996) have observed rapid variations of the Iron line profile and intensity in 1 to $10 \mathrm{ksec}$ in MCG-6-30-15 on the basis of an ASCA study. But this result has been recently questioned by Lee et al. (1999) and Chiang et al. (2000) who have studied with RXTE the spectral variability of MCG-6-3015 and of NGC 5548, and have shown that the $\mathrm{Fe} \mathrm{K}_{\alpha}$ line flux is constant over time scales of 50 to $500 \mathrm{ksec}$, while the underlying continuum displays large flux and spectral variations, and the profile of the line is variable! The same result seems to be observed in NGC 3615. Reynolds (2000) extended the work of Lee et al. (1999) on MCG-6-30-15, excluding a time delay between the line and the contin- uum in the range 0.5 to $50 \mathrm{ksec}$, and suggesting that the line flux remains constant on timescales between 0.5 and $500 \mathrm{ksec}$. From an analysis of the ASCA archive data of a sample of Seyfert nuclei, Weaver et al. (2001) found that in most cases changes in the line do not appear to track changes in the continuum.

These problems indicate, that either the geometry of the accretion flow envisioned as an X-ray lamp above the flat disk plane is not correct, or the response of the material is not as expected from simple estimates, or both.

In the present paper we concentrate on the second problem, i.e. on an accurate description of the X-ray reprocessing by an optically thick material. Since we do not want to restrict ourselves to a particular model of accretion flow we discuss two basic general cases: we consider a medium at constant pressure (with the density gradient determined by the temperature profile and the radiation plus gas pressure gradient) and we compare this situation to the case of a medium at constant density. We assume a plane parallel geometry. In the next paper we will explore the influence of the geometry, with particular attention given to a quasi-spherical case.

The radiative transfer computations are done with the coupled codes TITAN and NOAR described in detail by Dumont et al. (2000; hereafter DAC). We present a sequence of models characterized by a broad range of values of the ionization parameter defined at the surface. We also study the effects of other parameters involved, like the shape of the incident radiation flux.

We show the broad band spectra, characterize their overall properties by several parameters frequently measured observationally, like the $\alpha_{\mathrm{ox}}$ index and the amount of reflection as seen in XSPEC analysis, and finally we discuss the results comparing these quantities to typical parameters in various types of AGN.

\section{Radiation transfer}

The code TITAN was developed for the purpose of radiative transfer computations in an optically thick irradiated medium. Basically similar codes were developed by Ross \& Fabian (1993), Nayakshin et al. (2000; hereafter NKK), Madej \& Röżańska (2000). Other codes either do not cover the optical/UV energy range (Życki et al. 1994), or do not include hard X-ray irradiation (Hubeny et al. 2001), or they include hydrogen and helium only (Sincell \& Krolik 1997).

The code TITAN, the code of Ross \& Fabian (1993) and the NKK code based on XSTAR include the non-LTE effects while Madej \& Röżańska (2000) still work in the LTE approximation. Both Ross \& Fabian (1993) and NKK include the effect of Comptonization on the spectral distribution of photons while TITAN considers only the effect of Compton heating/cooling in energy balance. In order to obtain the Comptonized spectrum, the transfer code TITAN has to be supplemented with the Monte Carlo code NOAR of DAC. The code of Ross \& Fabian (1993), adopted by Ballantyne et al. (2001), contains only a small number 
of basis atomic lines. The major difference of TITAN with other codes (except that of Hubeny) is that it solves the line transfer instead of using the escape probability approximation.

The detailed description of the code TITAN and exemplary results for the temperature profile, ionization stage profile and the spectra in case of constant density of the medium are given by DAC and in Dumont \& Collin (2001). The code was already used to reproduce the mean quasar spectra within the frame of the cloud model (Czerny \& Dumont 1998), to model the iron line (after supplementing code results with NOAR: Abrassart 2000; Abrassart \& Dumont 1998, 2000; Karas et al. 2000) and to model the reflection by an accretion disk (after coupling with the computations of the hydrostatic equilibrium: Różańska et al. 2002).

\section{Results}

Since the dynamics of the formation of the hot and cold medium close to a black hole is not known it is necessary to reduce the problem to the consideration of a few basic possibilities for the description of the irradiated medium.

If the cold medium consists of clumps of cold material formed through a violent disruption of the disk accretion flow, the clouds may not reach an equilibrium and may be represented as a constant density medium. However, if the clouds are illuminated and can survive long enough, the medium will approach pressure equilibrium and an assumption of constant pressure may be a better representation of the medium, with the density gradient maintained due to the temperature and radiation plus gas pressure gradient. Finally, if the disk is not disrupted and it extends down to the marginally stable orbit (and possibly below it) the irradiated upper layers of the disk will be mostly in hydrostatic equilibrium. The last possibility is characterized by the steepest density gradient.

In the present paper we concentrate on the intermediate case, i.e. the medium in pressure equilibrium. We assume a plane-parallel geometry, and the medium is irradiated by an isotropic incident flux. The irradiated layers expand, and in the equilibrium situation a constant pressure is maintained throughout the entire zone. This pressure includes both the gas pressure and the radiation pressure.

\subsection{Constant pressure models}

We show first a set of models parameterized by the value of the ionization parameter $\xi$ at the surface of the irradiated medium $(z=0)$. This parameter is defined as

$\xi=\frac{L_{\mathrm{bol}}}{n(z=0) R^{2}}$,

where $L_{\mathrm{bol}}$ is the bolometric luminosity of the source located at a distance $R$ and $n(z=0)$ is the number density at the surface of the medium. In our computations the density was fixed at the value $n(0)=10^{11} \mathrm{~cm}^{-3}$.
Radiation pressure is locally calculated from

$P_{\mathrm{rad}}=\frac{4 \pi}{3 c} \int J_{\nu} \mathrm{d} \nu$

where $J_{\nu}$ is the mean intensity at a given depth computed at each step by the code.

The value of the total pressure (i.e. radiation + gas pressure) is calculated when the temperature of the plasma has been determined by the thermal balance equation, and this value is kept fixed within the medium thus determining the density profile. The model is iterated until convergence of the flux (and therefore of the radiation pressure) as in DAC.

Our basic sequence of models is specified as follows.

The shape of the incident radiation was assumed to be a power law, with the energy index $\alpha=1.0$, extending from $0.1 \mathrm{eV}$ to $100 \mathrm{keV}$.

The total hydrogen column in these computations is fixed at $3 \times 10^{25} \mathrm{~cm}^{-2}\left(\tau_{\mathrm{Th}} \approx 24\right)$.

The set of models with $\xi=300,10^{3}, 10^{4}, 10^{5}$ and $10^{6}$ illustrates the effect of increasing the incident flux from $2.7 \times 10^{12} \mathrm{erg} \mathrm{s}^{-1} \mathrm{~cm}^{-2}$ to $8 \times 10^{15} \mathrm{erg} \mathrm{s}^{-1} \mathrm{~cm}^{-2}$ correspondingly.

In all cases the irradiation results in the formation of a hot surface zone with an almost constant temperature. Initially the temperature decreases inwards rather slowly, with a rapid drop by 2 or 3 orders of magnitude below a certain depth.

This rapid transition reflects the ionization instability which develops in an irradiated medium (Krolik et al. 1981), and it leads to a temperature discontinuity if the medium is supposed to be in hydrostatic equilibrium (Begelman et al. 1983; Różańska \& Czerny 1996; Ko \& Kallman 1994; NKK). Unique, continuous solutions are obtained only if energy transfer by conduction is included, in addition to radiative heating and cooling (Begelman \& McKee 1990; Różańska 1999). However, at present no computations include both the full radiative transfer and the conduction term; either radiative transfer is replaced by a parametric description of heating and cooling (Różańska 1999) or the radiative transfer is computed carefully but the conduction is neglected (NKK, Ballantyne et al. 2001; Różańska et al. 2002 and other papers presenting spectra). In the second approach the instability is dealt with, either by adopting a discontinuity at an arbitrary position somewhere inside the multiple solution zone, or the smooth solution is found at the expense of a small pressure inversion in the multiple solution zone (see detailed discussion of this issue by Różańska et al. 2002). The conditions for the existence of multiple solutions under various approaches to line heating and cooling will be discussed by Coupé et al. (in preparation). In the present paper we adopt the continuous solution approach, as in Różańska et al. (2002).

We see in Fig. 1 (panel a) that both the surface temperature and the optical thickness of the hot layer depend strongly on the incident flux. Only the outermost layers of $\tau_{\text {es }} \sim 0.3$, and only at high irradiation flux, reach 

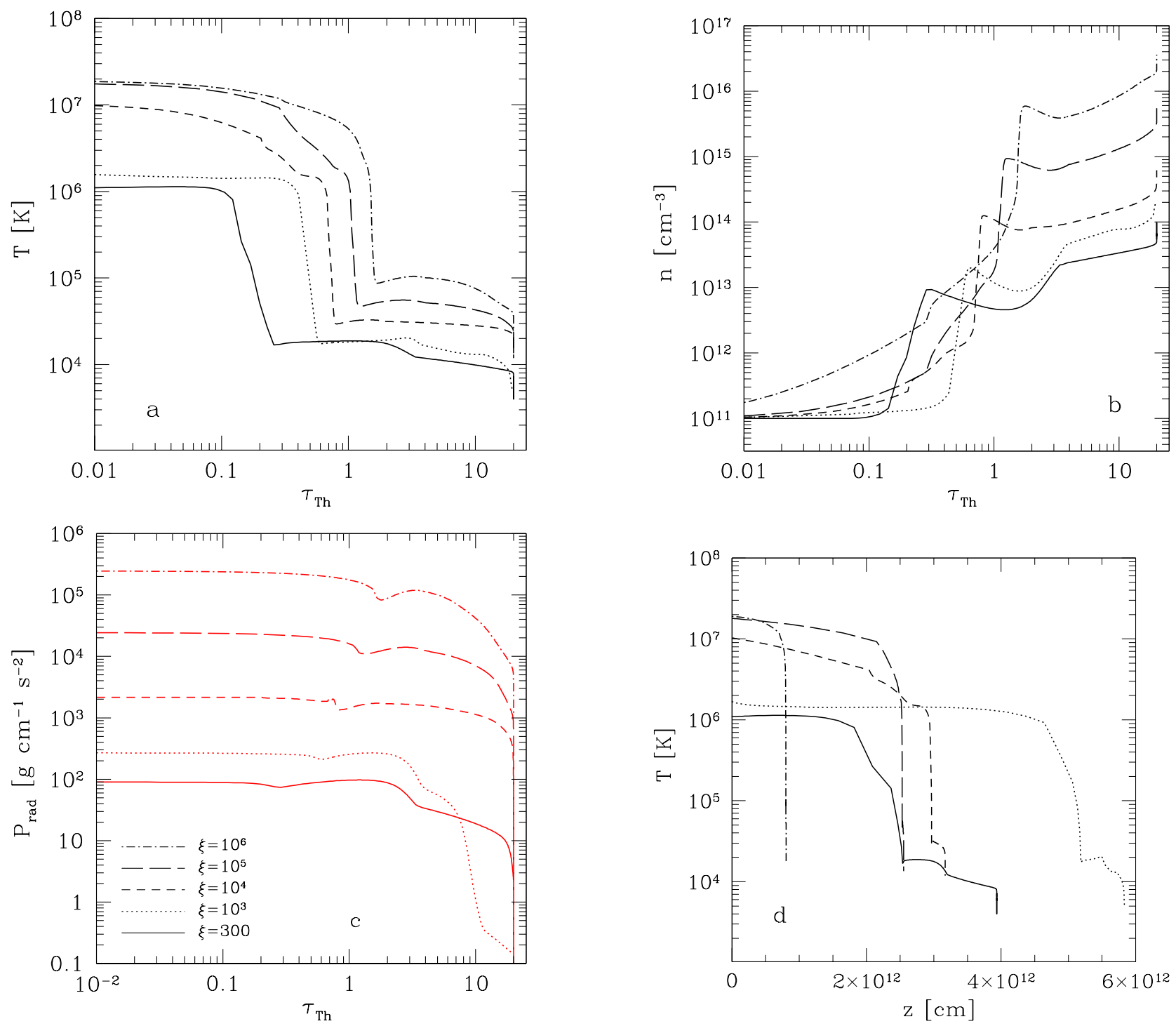

Fig. 1. The temperature (panel a)), density (panel b)) and pressure (panel c)) profiles as a function of the optical depth in Thomson units, in a constant pressure medium, for an ionization parameter at the surface $\xi=300,10^{3}, 10^{4}, 10^{5}$ and $10^{6}$ (continuous, dotted, short-dashed, long-dashed and dash-dot line, correspondingly); the density at the surface is $n(0)=$ $10^{11} \mathrm{~cm}^{-3}$, the column density is $N_{\mathrm{H}}=3 \times 10^{25} \mathrm{~cm}^{-2}$. The temperature profile as a function of the distance from the surface is shown in panel $\mathbf{d}$ ).

the Inverse Compton temperature, $T_{\mathrm{IC}}$, corresponding to the incident spectrum $\left(1.8 \times 10^{7} \mathrm{~K}\right)$, mostly because of the contribution coming from the thermalized back side radiation. For $\xi \leq 10^{3}$ the surface temperature is much lower than $T_{\mathrm{IC}}$ since the ionization of the upper layers is not high enough to make the Compton heating/cooling to dominate. The optical depth of the hot zone ranges from $\tau_{\mathrm{h}} \sim 0.6$ in the low flux case to $\tau_{\mathrm{h}} \sim 2$. Still lower ionization flux would lead to still lower optical depth of the hot skin and even lower surface temperature value. We do not show an example since the temperature of the back side of the slab approaches low values $\left(T \leq 10^{4} \mathrm{~K}\right)$ for which the code is not adapted.

As we assume a constant pressure the density profile results from the computations (see Fig. 1, panel b). The density contrast between the illuminated and back sides of the slab is very high since it reflects the temperature gradient as well as the considerable contribution from the radiation pressure which, in the case of high $\xi$, dominates the gas pressure by several orders of magnitudes in the hot layers (see Fig. 1, panel c).

This density gradient means that, while the cold region dominates the medium in terms of optical depth, the opposite is true if the spatial extension of the medium is considered. For example, for $\xi=10^{3}$ the hot region is $5 \times 10^{13} \mathrm{~cm}$ deep and the cold region is $10^{13} \mathrm{~cm}$, while for $\xi=10^{6}$ the hot region is $8 \times 10^{11} \mathrm{~cm}$ and the cold region only $4 \times 10^{9} \mathrm{~cm}$ ! Of course the cold medium can be geometrically and optically thicker but we do not consider larger values of the column density since that would not change the reflected spectra. Therefore, apart from the temperature plots as function of the optical depth we also 
plot the temperature against the true distance from the irradiated surface (see Fig. 1, panel d).

The large relative geometrical size of the heated layer has an important consequence in the case of the special geometry of a quasi-spherical distribution of clouds irradiated by the central source, as discussed by Collin-Souffrin et al. (1996). If the clouds have time to reach pressure equilibrium their irradiated parts would expand enormously and the medium will actually look more like clouds embedded in a warm bath, particularly for high irradiation flux. This will be particularly important when we will discuss the spherical geometry (Dumont et al., in preparation).

As we mentioned earlier, the ionization of iron is complete only close to the surface, and only in the case of the highest irradiation flux. Even for $\xi=10^{6}$ a large part of the hot layer is only partially ionized and populated with hydrogen and helium-like iron (see Fig. 2). The solution for $\xi=10^{3}$ is still mostly populated by FeXXV. Only in the case of the lowest value of the ionization parameter the contribution from highly ionized iron becomes negligible and the line is dominated by the $6.4 \mathrm{keV}$ component. In all other cases the iron line consists always of a number of components at various energies which reflect the thermal stratification of the medium.

The reflection spectra are given in Fig. 3. The thermalized fraction of the incident radiation decreases with increasing $\xi$ because the reflected fraction increases, due to the increased value of the temperature and of the optical depth of the hot layer, and the temperature of the UV bump increases because the temperature of deep layers increases. The Big Blue Bump due to the thermalized radiation is rather narrow and it never extends to the observed soft $X$-ray band. The same effect occurs in the case of a medium in hydrostatic equilibrium (NKK, Różańska et al. 2002). In the far UV band the Lyman edge can be seen in emission (for low values of $\xi$ ) or weakly in absorption (for $\xi \geq 10^{5}$ ). This is simply due to the fact that the underlying continuum becomes more intense in this band as $\xi$ increases. Soft X-ray lines superimposed on a reflected continuum are clearly visible in all cases. The continuum itself clearly shows the signatures of the ionized reflection since it is relatively flat on $\nu F_{\nu}$ plot and slightly rising below $2 \mathrm{keV}$, although not as strongly as in the constant density solutions (cf. Sect. 3.2).

The iron $K_{\alpha}$ line in all models consists of several components. The neutral component dominates only for $\xi=300$. In all other cases the line comes mostly from ionized iron ions. The helium-like iron contribution dominates for $\xi=10^{3}$, and the role of the hydrogen-like iron increases with further increase of $\xi$. This is not surprising if we consider the stratification of iron ion populations (see Fig. 2). The details of the spectra in this energy band determined from NOAR are shown in Fig. 4. Equivalent widths of the line components determined with respect to the total (i.e. reflected plus incident) radiation flux are given in Table 1.

The line is broadened by Comptonization and a red tail of the line develops for large values of the ionization
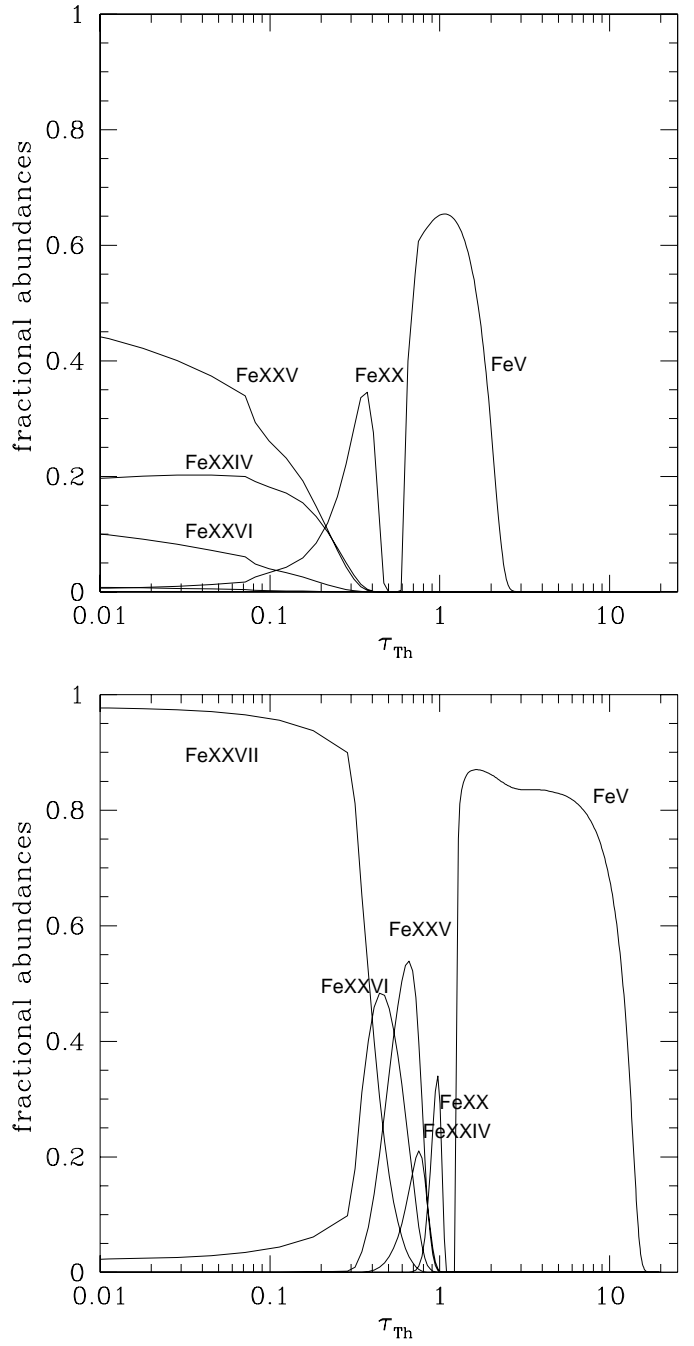

Fig. 2. The population of iron ions as a function of optical depth in a constant pressure medium, for $\xi=10^{3}$ (upper panel) and $10^{5}$ (lower panel); $n(0)=10^{11} \mathrm{~cm}^{-3}, N_{\mathrm{H}}=3 \times 10^{25} \mathrm{~cm}^{-2}$.

parameter. A narrow core, or rather several narrow components remain, and the effect of Comptonization can be seen as an additional broad underlying component.

\subsection{Comparison with a constant density case}

The reflection from the constant density medium was already broadly discussed in the literature (Ross \& Fabian 1993; Życki et al. 1994; Dumont \& Collin 2001), including the Fe $\mathrm{K}_{\alpha}$ line properties (Matt et al. 1993; Życki \& Czerny 1994). However, the comparison is much easier if the computations are made with the same code and for the same sets of corresponding parameters.

Therefore we show two examples, computed for an incident power law spectrum, the density equal to $10^{11} \mathrm{~cm}^{-3}$ everywhere in the medium and the total column density to $3 \times 10^{25} \mathrm{~cm}^{-2}$ as before.

The temperature profile (compare Figs. 1 and 5) is much more shallow that in the constant pressure case, the heated zone is effectively much broader. The overall 


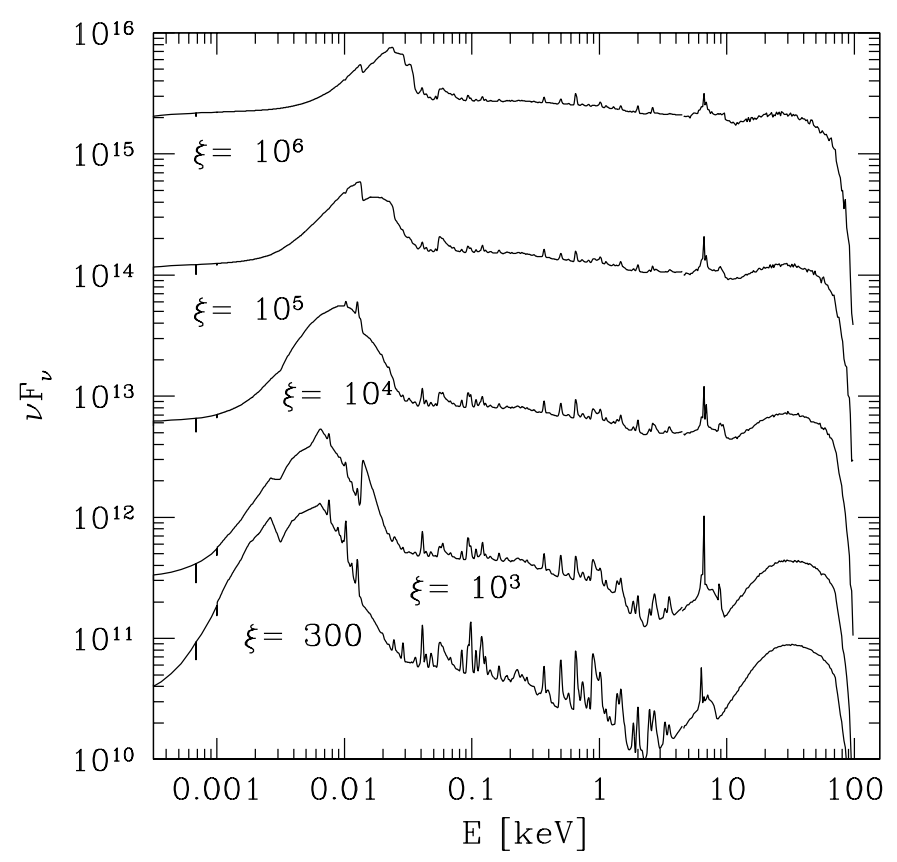

Fig. 3. The reflection component from a constant pressure medium for $\xi=300,10^{3}, 10^{4}, 10^{5}$ and $10^{6} ; n(0)=10^{11} \mathrm{~cm}^{-3}$, $N_{\mathrm{H}}=3 \times 10^{25} \mathrm{~cm}^{-2}$. Spectral resolution: 30 .

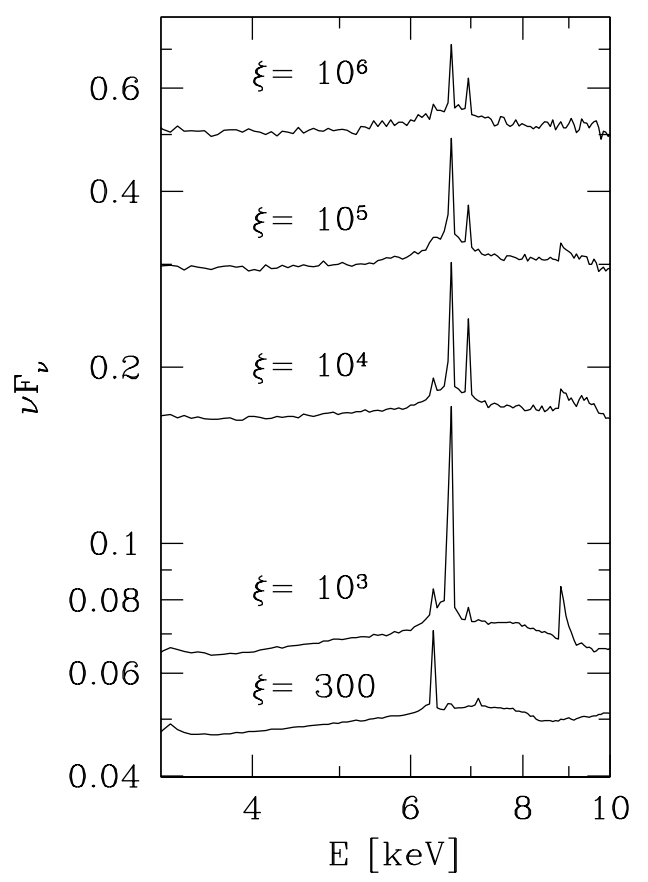

Fig. 4. The total spectrum (reflection plus primary) in arbitrary units in the region of the iron line, computed with the NOAR code for a constant pressure medium; $\xi=300,10^{3}$, $10^{4}, 10^{5}$ and $10^{6} ; n(0)=10^{11} \mathrm{~cm}^{-3}, N_{\mathrm{H}}=3 \times 10^{25} \mathrm{~cm}^{-2}$. Resolution: 100.

spectrum (see Fig. 6) varies much more strongly with the incident flux than in the constant pressure case, and displays a much broader "Big Blue Bump", due to the presence of layers with intermediate temperature. This strong, almost a power law, tail extends up to $2 \mathrm{keV}$ thus forming a strong soft X-ray excess.

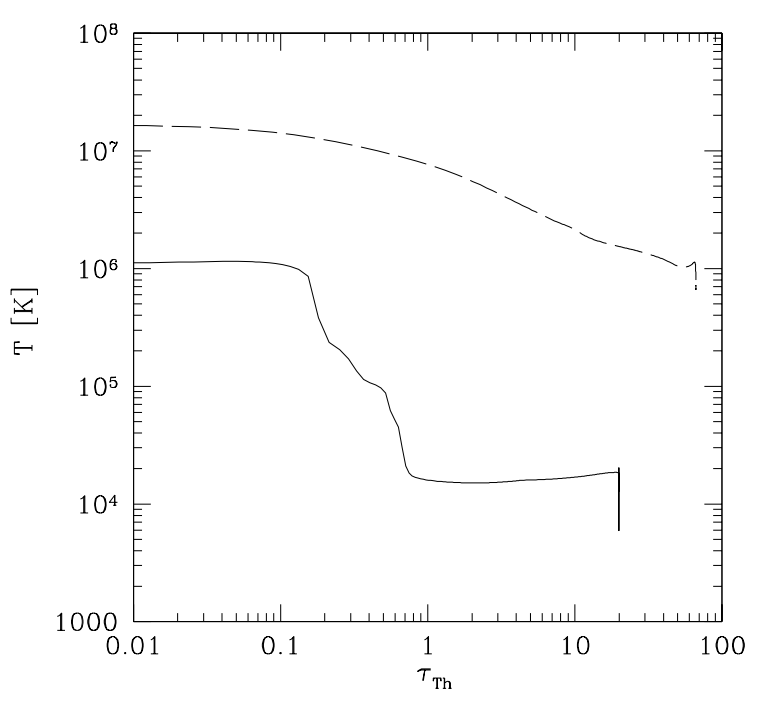

Fig. 5. The temperature profile in a constant density medium for $\xi=300, N_{\mathrm{H}}=3 \times 10^{25} \mathrm{~cm}^{-2}$ (solid line), and for $\xi=10^{5}$; $N_{\mathrm{H}}=10^{26} \mathrm{~cm}^{-2} ; n(0)=10^{11} \mathrm{~cm}^{-3}$ (dotted line).

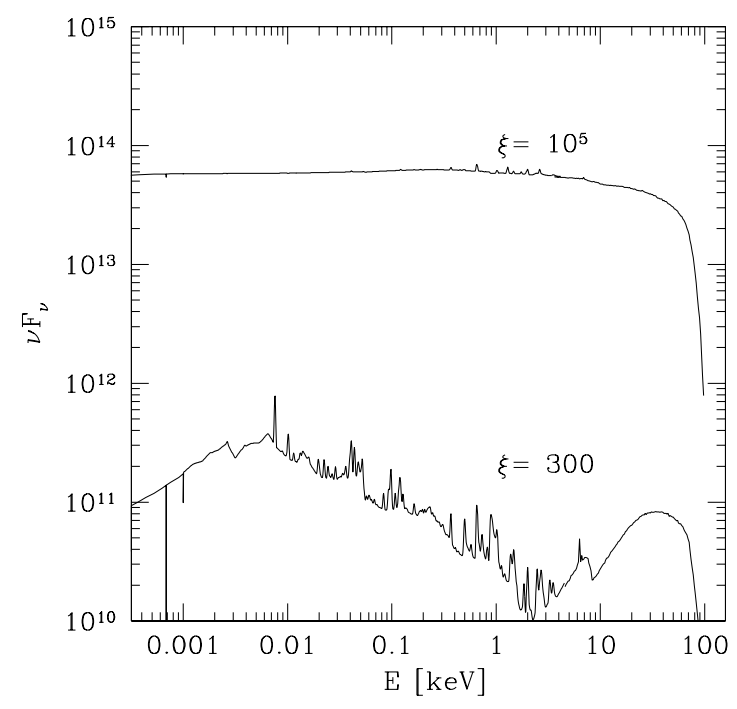

Fig. 6. The reflection component from a constant density medium, for $\xi=300, N_{\mathrm{H}}=3 \times 10^{25} \mathrm{~cm}^{-2}$, and for $\xi=10^{5}$; $N_{\mathrm{H}}=10^{26} \mathrm{~cm}^{-2} ; n(0)=10^{11} \mathrm{~cm}^{-3}$. Resolution: 30 .

Despite the more shallow temperature profile the iron line in the constant density case is usually dominated by a single component. This is due to the fact that the zone where the temperature traces the ionization state is Thomson thick and the line is effectively dominated by the ionization state of the outer layers thus giving a cold line for low $\xi$, and $6.7-6.9 \mathrm{keV}$ lines for higher irradiation flux. Any particular ionization stage of the iron can be modeled by adjusting the incident flux, which is not the case either in constant pressure computations or in solutions in hydrostatic equilibrium, as noticed by NKK.

In the case of high ionization parameter $\left(\xi=10^{5}\right)$ the line again is broadened by Compton downscattering (see Fig. 7) and the effect is stronger in the constant density medium than in the constant pressure case (see also Abrassart \& Dumont 2000 and Abrassart 2000). In the 


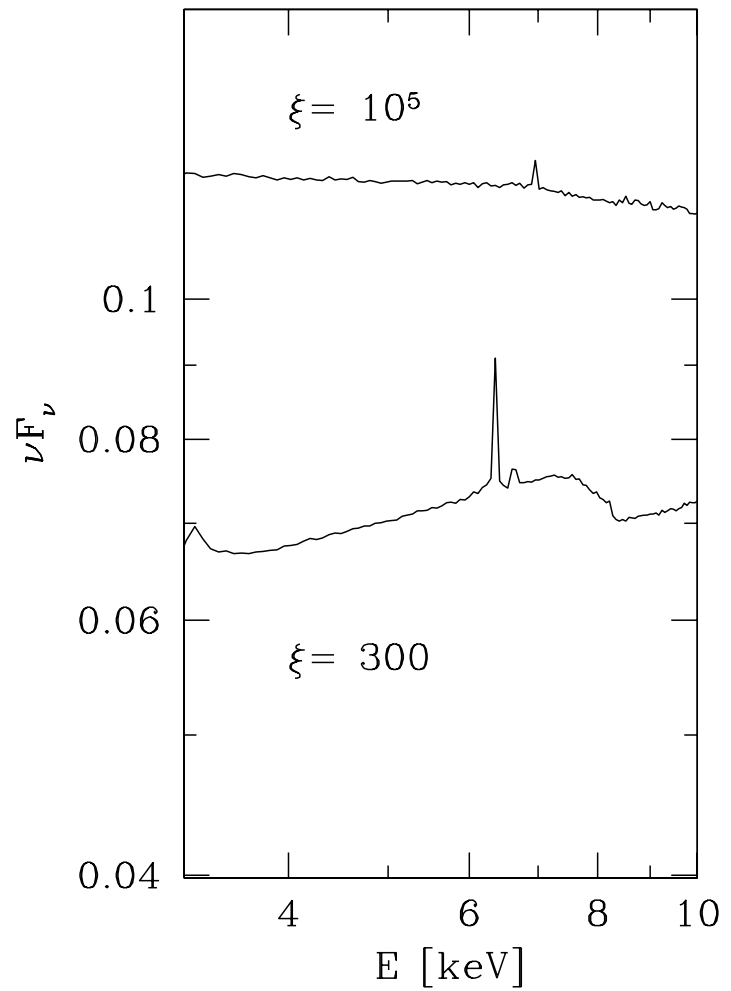

Fig. 7. The total spectrum (reflection plus primary) in arbitrary units in the region of the iron line, computed with the NOAR code for a constant density medium; $\xi=300$, $N_{\mathrm{H}}=3 \times 10^{25} \mathrm{~cm}^{-2}$, and for $\xi=10^{5} ; N_{\mathrm{H}}=10^{26} \mathrm{~cm}^{-2}$; $n(0)=10^{11} \mathrm{~cm}^{-3}$. Resolution: 100 .

case of low $\xi$ the line is dominated by the narrow cold component but we also see a strong absorption edge. Such a feature is only barely seen from the constant pressure medium.

\subsection{Comparison with hydrostatic equilibrium}

Two examples of reprocessing by an irradiated accretion disks were presented by Różańska et al. (2002). The parameterization of such models is through the accretion rate, and the pressure increases towards the disk equatorial plane. However, we can compare two models with similar total pressure at the surface.

In the model computed for a black hole mass $M=$ $10^{8} M_{\odot}$, an accretion rate $\dot{m}=0.03 \dot{M}_{\text {Edd }}$ and a distance $10 R_{\mathrm{Schw}}$, the gas pressure is of the order of $2 \times 10^{3} \mathrm{~g} \mathrm{~cm}^{-1} \mathrm{~s}^{-2}$ in the surface layers, so it approximately corresponds to our case $\xi=10^{4}$ of constant pressure models.

The surface temperature is slightly lower in the constant pressure model since the slope of the incident radiation is different in the two models ( $\alpha=0.9$ was adopted in the hydrostatic equilibrium model, while $\alpha=1.0$ in the present computations). The overall shape of the reflected spectrum in X-ray band is similar, apart from the effect caused by the difference in the adopted incident flux in the two computations. The iron $\mathrm{K}_{\alpha}$ line in both cases shows all three components $(6.4 \mathrm{keV}, 6.7 \mathrm{keV}$ and $6.9 \mathrm{keV})$, but the neutral component is relatively stronger in the case of hydrostatic equilibrium. The Big Blue Bump peak is also relatively stronger in the case of hydrostatic equilibrium. This is partially due to an additional energy generation in the accretion disk itself and partially due to a much thicker hot skin in the case of constant pressure medium $(\sim 0.7$ instead of $\sim 0.1$ ) which increases the reflected fraction at the expense of the reprocessed one. Increased Thomson thickness of the radiatively heated zone accounts also for the difference in the value of the equivalent width of the iron $\mathrm{K}_{\alpha}$ line - the line from the constant pressure model is by $50 \%$ weaker than in the hydrostatic equilibrium case (66 eV vs. $92 \mathrm{eV}$ ).

Similar differences are seen between the irradiated disk model $\dot{m}=0.3$ (hydrostatic equilibrium) and the (constant pressure) model with $\xi=10^{5}$, both having a total surface pressure of order of $2 \times 10^{4}$. Also in this case the skin is thicker in the constant pressure case $\left(\tau_{\mathrm{Th}}=1 \mathrm{vs}\right.$. $\tau_{\mathrm{Th}}=0.3$ ), and consequently the equivalent width of the $\mathrm{Fe} \mathrm{K}_{\alpha}$ line is lower ( $80 \mathrm{eV}$ in hydrostatic equilibrium case and $53 \mathrm{eV}$ in constant pressure case), and the dominance of the $6.7 \mathrm{keV}$ component in the line profile is much stronger in the constant pressure solution.

\subsection{The effect of the spectral shape of incident radiation}

\subsubsection{Power law slope}

The slope of the incident radiation is important not only because of its contribution to the observed spectrum. It also modifies the spectral shape of the reflected component by affecting the value of the Compton temperature and the thickness of the hot layer.

In constant density computations the incident slope determines the reflected slope in the soft part of the spectrum (see Fig. 6 of Czerny \& Dumont 1998) - a steeper incident flux results in a proportionally steeper reflected component. The properties of the iron line do not change dramatically.

A medium in hydrostatic equilibrium reacts differently to such changes (NKK): a harder spectrum results in a completely ionized outer skin and a relatively faint but neutral iron line produced in the deeper un-ionized zone, while a softer spectrum gives only a partially ionized skin and a strong iron line coming from ionized iron ions in that region.

The reaction of a medium at constant pressure is intermediate between these two cases (see Fig. 8). When the radiation spectrum is very hard $(\alpha=0.7)$ the dominant contribution to the line comes indeed from the deep layers and the $6.4 \mathrm{keV}$ component dominates. Intermediate slopes enhance the contribution from helium-like iron and both line components (6.4 and $6.7 \mathrm{keV}$ ) are clearly seen. However, when the spectrum is very soft $(\alpha=1.3)$, a cold iron line dominates again since the gas is not heated to a high enough temperature (even at the surface) for highly ionized iron to contribute. 


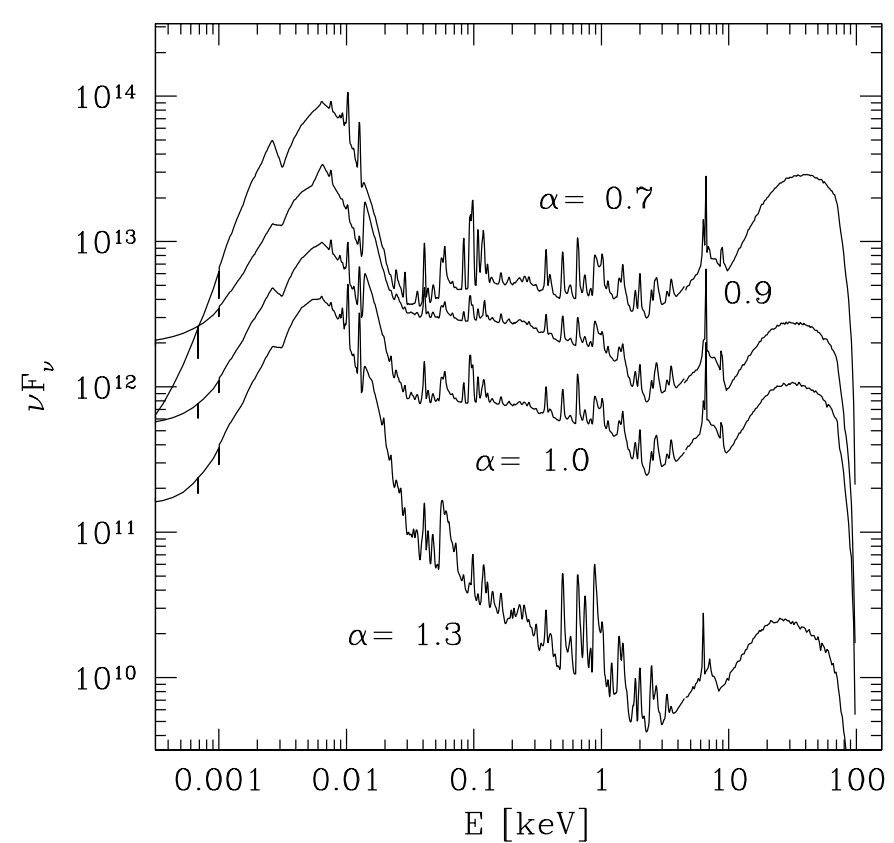

Fig. 8. The dependence of the reflection component from a constant pressure medium on the slope of the incident radiation spectrum $\alpha$ equal $0.7,0.9,1.0$ and 1.3 , for $\xi=10^{3} ; n(0)=$ $10^{11} \mathrm{~cm}^{-3}, N_{\mathrm{H}}=3 \times 10^{25} \mathrm{~cm}^{-2}$. Spectra are shifted for better illustration. Resolution: 30 .

\subsubsection{The spectrum of Laor et al. (1997)}

A hot (thermal) plasma is a source of a power law radiation which is formed due to Compton upscattering of the soft photons by energetic electrons. A cold layer thermalizes a fraction of the radiation but nevertheless, in the plane-parallel geometry, the X-ray primary emission dominates the total spectrum. This is not a good representation of high accretion rate objects like quasars or NLS1 galaxies (where the soft thermal component dominates), which means that either the geometry is different, or most of the energy is dissipated within the cold phase at larger distances from the black hole. In this latter case the considered innermost cold layer will see not only the hard $\mathrm{X}$-ray source but it will be also affected by the mean radiation field.

To illustrate this situation we consider the case of a layer irradiated by a source with a spectral distribution given by the mean spectral shape of quasars, as determined by Laor et al. (1997) from a large quasar sample. This radiation field is much softer than our assumed power law, since it is strongly dominated by the Big Blue Bump, and the corresponding Inverse Compton temperature is lower than for a power law spectrum with $\alpha=1.0$ $\left(2.4 \times 10^{6} \mathrm{~K}\right.$ and $1.9 \times 10^{7} \mathrm{~K}$, correspondingly).

The reflected spectrum in the case of a constant pressure medium is shown in Fig. 9. It traces to some extent the incident spectrum, giving a strong soft X-ray excess, unlike in the case of an incident power law spectrum. The overall shape is much more similar to the case of constant density computations, with a power law spectrum. A strong soft X-ray excess is clearly seen below $2 \mathrm{keV}$.

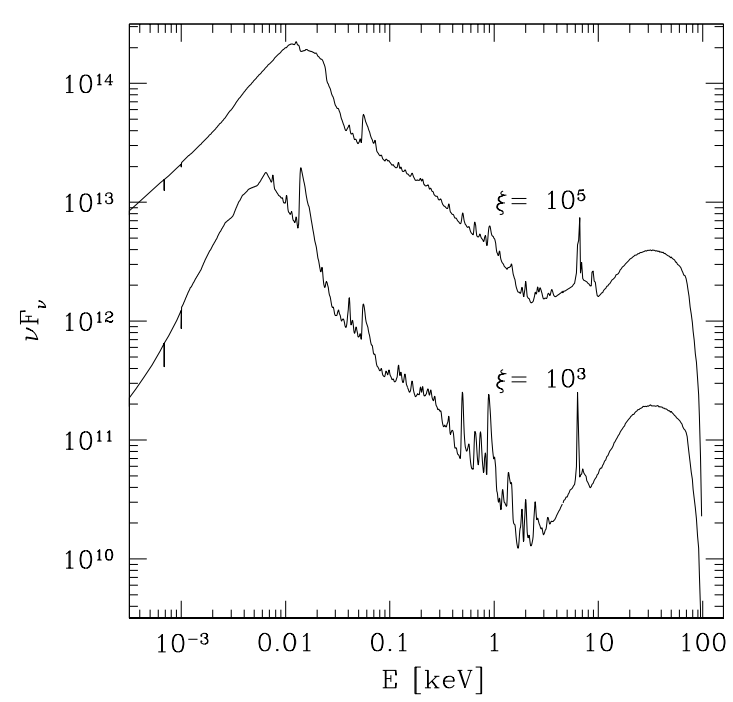

Fig. 9. The reflection component from a constant pressure medium for the incident radiation spectrum given by Laor et al. (1997), for $\xi=10^{3}$ and $10^{5}$. Parameters: $n(0)=10^{11} \mathrm{~cm}^{-3}$, $N_{\mathrm{H}}=3 \times 10^{25} \mathrm{~cm}^{-2}$. Resolution: 30 .

In the $\mathrm{K}_{\alpha}$ line profile a strong contribution from $6.4 \mathrm{keV}$ component is always seen, although for $\xi \leq 10^{5}$, the $6.7 \mathrm{keV}$ component is slightly stronger (see Fig. 10). A model with a power law incident radiation and a comparable hard X-ray flux does not show such a strong neutral component.

\subsection{Total column density}

Our computations were deliberately made for a Thomson thick medium. However, an interesting question is, how strong the effect of the column density is for a moderately thick medium, and what optical thickness of the medium is needed to thermalize the incident radiation.

In the case of a constant density medium the photons penetrate quite deeply into the medium if the irradiation is strong. We saw in Fig. 5 that for $\xi=10^{5}$ the temperature of the back side was still quite high even for the very large column density adopted in this computation $\left(10^{26} \mathrm{~cm}^{-2}\right)$.

However, in constant pressure medium the thermalization is much more efficient for the same Thomson optical depth due to the fact that the density is much higher in the cold region, and therefore collisional cooling is much more efficient. We give two examples of the reflection spectra calculated for a moderate value of the ionization parameter $\left(\xi=10^{3}\right)$ and two values of the column density (corresponding to $\tau_{\mathrm{Th}}=20$ and $\tau_{\mathrm{Th}}=2$; Fig. 11). Even if the total optical thickness of the zone is equal to 2 the temperature at the back side of the layer is low. The resulting spectra are almost indistinguishable.

\subsection{Density}

Parameterization of the incident flux with the ionization parameter $\xi$ has the advantage of reducing significantly 


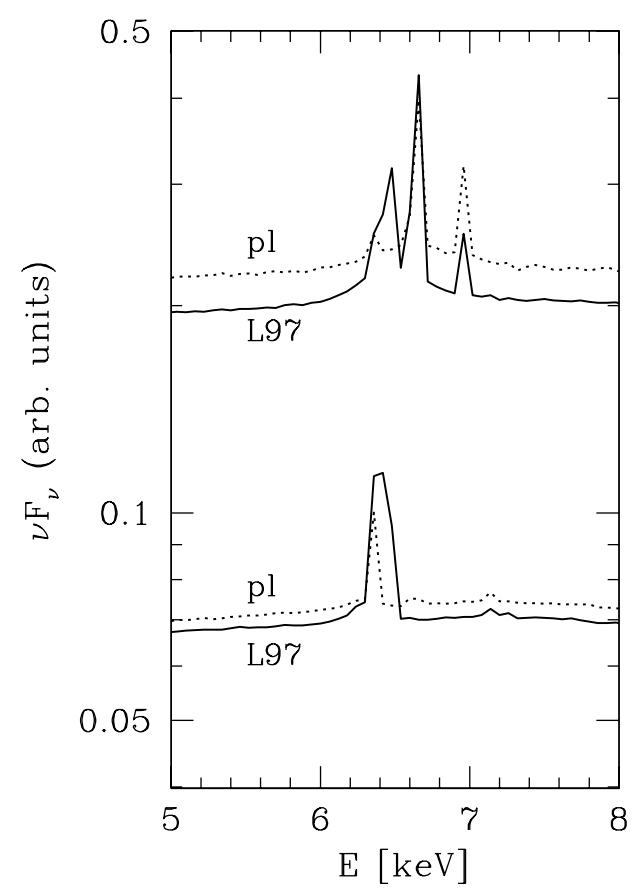

Fig. 10. The total spectrum (reflected plus primary) in the region of the iron $\mathrm{K}_{\alpha}$ line for two sets of models, with similar normalization of the incident flux at $2 \mathrm{keV}$ but two shapes of the incident radiation spectra. First set: power law spectrum with $\xi=300$ and the Laor et al. (1997) model with $\xi=10^{3}$. Second set: power law spectrum with $\xi=10^{4}$ and the Laor et al. (1997) spectrum with $\xi=10^{5}$ from a constant pressure medium. Other parameters: $n(0)=10^{11} \mathrm{~cm}^{-3}, N_{\mathrm{H}}=3 \times$ $10^{25} \mathrm{~cm}^{-2}$. Resolution: 100 .

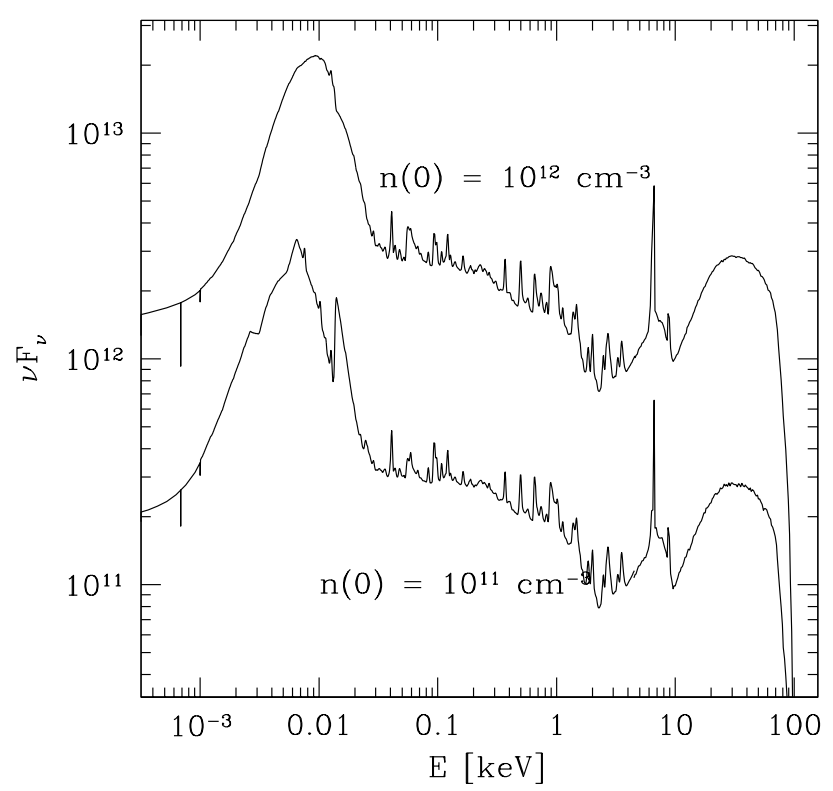

Fig. 11. The reflection component from a constant pressure medium for $\xi=10^{3} ; n(0)=10^{11} \mathrm{~cm}^{-3}$, and two values of the column density: $N_{\mathrm{H}}=3 \times 10^{25} \mathrm{~cm}^{-2}$ and $N_{\mathrm{H}}=3 \times 10^{24} \mathrm{~cm}^{-2}$. The spectra are shifted for a better illustration. Resolution: 30 .

the dependence on the medium density. In constant density computations this approach was particularly succesfull and the reflection spectra do not depend significantly

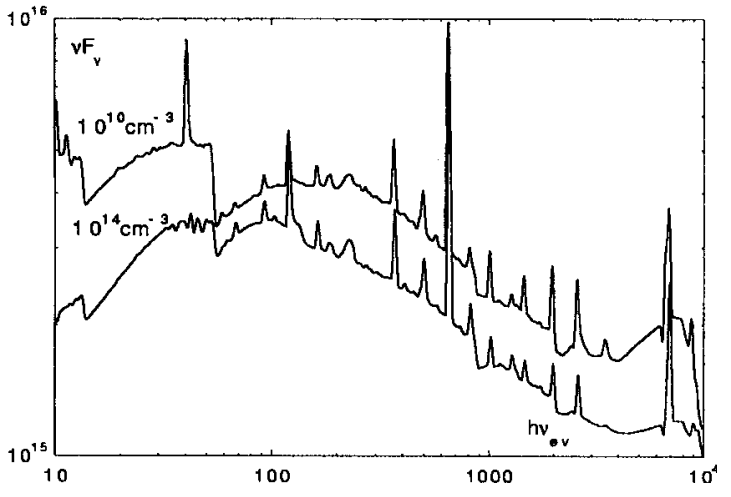

Fig. 12. The reflection component from a constant density medium for the power law incident radiation spectrum for $\xi=$ $10^{3}$ and two values of the number density; $n(0)=10^{10} \mathrm{~cm}^{-3}$ and $n(0)=10^{14} \mathrm{~cm}^{-3} ; N_{\mathrm{H}}=3 \times 10^{25} \mathrm{~cm}^{-2}$. The spectra are shifted for a better illustration. Resolution: 30 .

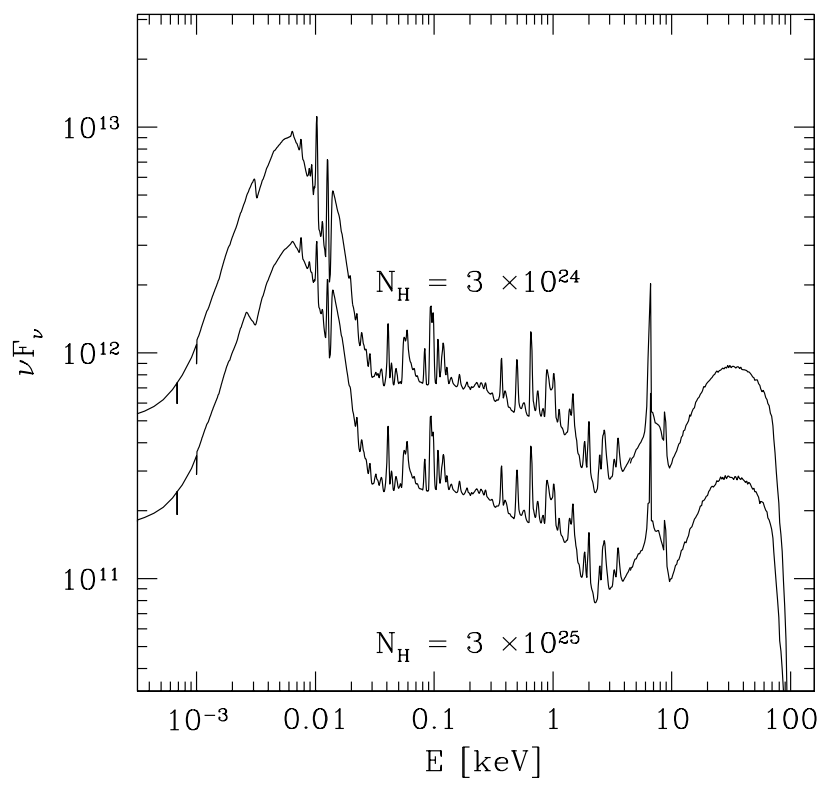

Fig. 13. The reflection component from a constant pressure medium for the power law incident radiation spectrum for $\xi=$ $10^{3}$ and two values of the number density; $n(0)=10^{11} \mathrm{~cm}^{-3}$ and $n(0)=10^{12} \mathrm{~cm}^{-3} ; N_{\mathrm{H}}=3 \times 10^{25} \mathrm{~cm}^{-2}$.

on the density in a broad range of parameters (from $n=10^{10} \mathrm{~cm}^{-3}$ up to $n=10^{14} \mathrm{~cm}^{-3}$ ). Some differences are seen in the low energy band (IR and optical) due to the influence of free-free emissivity and the reflection hump above $\approx 3 \mathrm{keV}$ is more prominent in the low density case (Fig. 12).

Radiation transfer computations performed in constant pressure medium are much more sensitive to the density itself due to the density gradient. When the surface density is changed the radiation to gas pressure ratio changes at the surface and the new density profile as a function of the optical depth changes, and it is not just a rescaled version of the old profile by a constant value. This induces some differences in the broad band spectrum as well as in details of the spectral features (see Fig. 13). 
Table 1. Summary of model properties.

\begin{tabular}{rrrrrrrrrrrr}
\hline No. & \multicolumn{1}{c}{$\xi^{a}$} & incid $^{b}$ & \multicolumn{1}{c}{$n^{c}$} & $N_{\mathrm{H}}^{d}$ & $E_{\max }^{f}$ & $\alpha_{\mathrm{Ox}}^{g}$ & $(6.4)$ & $(6.7)$ & $(6.9)$ & $R_{\text {XSPEC }}^{h}$ & $\Gamma^{j}$ \\
\hline \hline constant pressure & & & & & & & & & \\
\hline 1 & $3 \times 10^{2}$ & 1.0 & $1 \times 10^{11}$ & $3 \times 10^{25}$ & 5.21 & 1.31 & 18.0 & 3.4 & 0.0 & $1.53 \pm 0.17$ & $2.09 \pm 0.05$ \\
2 & $1 \times 10^{3}$ & 1.0 & $1 \times 10^{11}$ & $3 \times 10^{25}$ & 6.45 & 1.25 & 14.9 & 96.7 & 5.7 & $1.82 \pm 0.10$ & $2.11 \pm 0.03$ \\
3 & $1 \times 10^{4}$ & 1.0 & $1 \times 10^{11}$ & $3 \times 10^{25}$ & 8.39 & 1.13 & 4.5 & 42.8 & 19.2 & $1.14 \pm 0.05$ & $2.06 \pm 0.02$ \\
4 & $1 \times 10^{5}$ & 1.0 & $1 \times 10^{11}$ & $3 \times 10^{25}$ & 12.62 & 1.04 & 5.8 & 37.2 & 9.7 & $0.88 \pm 0.08$ & $2.05 \pm 0.02$ \\
5 & $1 \times 10^{6}$ & 1.0 & $1 \times 10^{11}$ & $3 \times 10^{25}$ & 22.68 & 1.01 & 2.9 & 18.8 & 8.8 & $0.58 \pm 0.02$ & $2.05 \pm 0.02$ \\
6 & $1 \times 10^{3}$ & 1.0 & $1 \times 10^{11}$ & $3 \times 10^{24}$ & 6.39 & 1.24 & 74.9 & 102.6 & 4.0 & $1.61 \pm 0.09$ & $2.10 \pm 0.03$ \\
7 & $1 \times 10^{3}$ & 0.7 & $1 \times 10^{11}$ & $3 \times 10^{25}$ & 2.37 & 1.16 & 16.0 & 78.7 & 4.2 & $1.71 \pm 0.07$ & $1.81 \pm 0.02$ \\
8 & $1 \times 10^{3}$ & 1.3 & $1 \times 10^{11}$ & $3 \times 10^{25}$ & 5.97 & 1.58 & 33.9 & 0.0 & 0.0 & $0.87 \pm 0.04$ & $2.06 \pm 0.03$ \\
9 & $1 \times 10^{3}$ & 1.0 & $1 \times 10^{12}$ & $3 \times 10^{25}$ & 19.64 & 1.11 & 87.0 & 99.8 & 2.1 & $1.83 \pm 0.03$ & $2.11 \pm 0.02$ \\
10 & $1 \times 10^{5}$ & Laor & $1 \times 10^{11}$ & $3 \times 10^{25}$ & 11.30 & 1.54 & 70.0 & 91.1 & 13.0 & $17.21 \pm 0.45$ & $2.77 \pm 0.05$ \\
11 & $1 \times 10^{3}$ & Laor & $1 \times 10^{11}$ & $3 \times 10^{25}$ & 6.50 & 1.66 & 114.5 & 0.0 & 0.0 & $17.95 \pm 0.55$ & $2.76 \pm 0.05$ \\
\hline \hline constant density & & & & & & & & & & \\
\hline 12 & $3 \times 10^{2}$ & 1.0 & $1 \times 10^{11}$ & $3 \times 10^{25}$ & 4.97 & 1.15 & 19.9 & 4.0 & 0.0 & $2.19 \pm 0.18$ & $2.12 \pm 0.01$ \\
13 & $1 \times 10^{5}$ & 1.0 & $1 \times 10^{11}$ & $1 \times 10^{26}$ & 368.6 & 0.99 & 0.0 & 0.0 & 1.0 & $0.15 \pm 0.06$ & $2.02 \pm 0.01$ \\
14 & $1 \times 10^{3}$ & 1.0 & $1 \times 10^{12}$ & $3 \times 10^{25}$ & 10.4 & 1.18 & 0.0 & 105.9 & 4.0 & $1.32 \pm 0.08$ & $2.03 \pm 0.03$ \\
15 & $3 \times 10^{2}$ & Laor & $1 \times 10^{14}$ & $3 \times 10^{25}$ & 9.86 & 1.55 & 89.8 & 0.0 & 0.0 & $11.13 \pm 0.01$ & $2.78 \pm 0.02$ \\
16 & $2 \times 10^{6}$ & Laor & $1 \times 10^{11}$ & $3 \times 10^{24}$ & 11.11 & 1.50 & 0.0 & 0.0 & 7.1 & $14.34 \pm 0.01$ & $2.69 \pm 0.02$ \\
\hline
\end{tabular}

a - Ionization parameter.

${ }^{b}$ - Incident spectrum shape (either AGN mean spectrum after Laor et al. 1997), or a power law with an energy index ( $\alpha$ ).

${ }^{c}$ - Number density at the surface.

${ }^{d}$ - Total column density of the medium.

$f$ - Energy at which the spectrum has its maximum at the EUVE range in $[\mathrm{eV}]$.

${ }^{g}$ - Effective spectral index measured between $2500 \AA$ and $2 \mathrm{keV}$.

$h$ - Amount of reflection as determined from PEXRAV XSPEC model.

$j$ - X-ray slope as determined from PEXRAV XSPEC model.

\subsection{Summary of models}

In order to facilitate the comparison between the various models presented here and the observed properties of the AGN spectra we determine for those models several broadly used quantities. The results are presented in Table 1 and they include more models than just those presented in the previous subsections.

Since the computations are made in plane-parallel geometry the final spectrum consists of the reflected spectrum plus the incident spectrum, without any additional weight.

We introduce the $\alpha_{\text {ox }}$ parameter as the mean slope calculated between $2500 \AA$ and $2 \mathrm{keV}$. We give the widths of the components of the $\mathrm{Fe} \mathrm{K}_{\alpha}$ line, measured with respect to the observed continuum (i.e. reflected plus incident) which result directly from our computations.

Since the observed spectra are usually analyzed using the XSPEC software (and the standard spectral components provided with it) we follow other authors presenting their theoretical results (Done \& Nayakshin 2001; Ballantyne et al. 2001) and we analyze our spectra as we would analyze an observed spectrum.

We create artificial data using the fake command in $\mathrm{XSPEC}$ for ASCA satellite and assuming the count rate at the typical level expected from bright AGN sources with a long exposure.

We fit such data using PEXRAV model (Magdziarz \& Zdziarski 1995) to represent the incident power law and the reflected continuum, and we add GAUSSIAN to account for the iron line. These fits provide us again with the normalization of the reflected component, $R_{\mathrm{XSPEC}}$, and the photon index of the incident radiation, $\Gamma$. In principle $R_{\text {XSPEC }}$ should be equal to 1 , while $\Gamma$ should be equal to the photon index adopted in our model computations. However, the shape of the reflected component assumed in PEXRAV is rather different from the one from our computations of radiative transfer and so both $R_{\mathrm{XSPEC}}$ and $\Gamma$ are affected. The results for various models are summarized in Table 1 . We see that low $\xi$ models show a reflection component larger than 1 , while reflection from highly ionized material give values significantly lower than 1 . This effect is much stronger in the constant density models which are characterized by a reflection coefficient of the order of unity for a rather narrow range of values of the ionization parameter. Fits to models with the Laor et al. (1997) incident spectrum gave formally very large values of the reflection coefficient which was caused by the presence of the strong soft X-ray excess in those spectra models. We did not add any additional component during the fitting 
to account for this excess which leads to an artificially steep "incident radiation" and a large reflection.

We also considered the XSPEC PEXRIV model (Magdziarz \& Zdziarski 1995) of ionized reflection. However, since the shape of the reflection component is different in our computations and in PEXRIV, this model did not provide a better description of our model and the returned value of the ionization parameter was usually rather low independently from the true value characterizing the model. Similar problems were found by Ballantyne et al. (2001).

\section{Discussion}

$\mathrm{X}$-ray reprocessing in plane-parallel geometry under the assumption of the constant density has been studied in a number of papers (Ross \& Fabian 1993; Życki et al. 1994; DAC; see Dumont \& Collin 2001). Similar computations were also performed for a stratified medium in hydrostatic equilibrium (Raymond 1993, NKK; Ballantyne et al. 2001; Różańska et al. 2002).

In the present paper we consider an intermediate case: the medium under constant pressure. It may represent a strongly flattened distribution of clumpy accretion flow.

Our model, as most of the constant density or hydrostatic models, is clearly oversimplified since we assume that the entire flow is characterized by a single value of the ionization parameter, which may not be the case in a real accretion flow. We also did not include here the dissipation which may take place in the cold phase of an accretion flow and consequently enhance the contribution of the Big Blue Bump to the overall spectrum. This last effect is only simulated to some extent by considering the shape of the incident radiation flux in the form of the mean quasar spectrum as determined by Laor et al. (1997).

Nevertheless, the results illustrate certain important trends which should help to analyse the conditions in the emitting plasma.

Below we compare the results obtained from the constant pressure medium with the observed trends in the broad band spectra of AGN.

\subsection{Observed $\alpha_{\text {ox }}$ properties in quasar and Seyfert galaxies samples}

The observed broad band spectral index $\alpha_{\text {ox }}$ measured between $2500 \AA$ and $2 \mathrm{keV}$ was shown for a sample of Seyfert galaxies by Walter \& Fink (1993) and for quasars by Green (1996). Both samples showed a large dispersion of this quantity, which could be partly explained by reddening (e.g. Wilkes et al. 1999; Laor \& Brandt 2002). The average values are about 1.3 for Seyferts and 1.5 for quasars, with most Seyferts having values between 1 and 1.6, and most quasars having values between 1.2 and 1.8.

Our constant pressure models with an incident power law radiation slope equal to 1 have on average somewhat lower values of the $\alpha_{\mathrm{ox}}$, around 1.2, with typical values between 1 and 1.3. Since the observed power law index in
Seyfert galaxies is on average not steeper than the value adopted in our computations it shows that either there is some dissipation within the cold medium which slightly contributes to the Big Blue Bump, or the plane-parallel geometry adopted in the computations is not correct (for spherical accretion models see Collin-Souffrin et al. 1996; Czerny \& Dumont 1998; and Malzac 2001). The effect is even stronger in the case of quasars. Not surprisingly, the models with an incident flux based on averaged quasar spectra reproduce the observed values of $\alpha_{\mathrm{ox}}$ for those models.

Hydrostatic equilibrium models give for $\alpha_{\mathrm{ox}}$ results comparable to the constant pressure models (see Różańska et al. 2002), with values slightly lower than seen in Seyfert galaxies, and lower than in quasars. Constant density models give still lower values $(\sim 1)$ and a bigger discrepancy between the simple prediction and the data.

\subsection{Amplitude of the reflection component}

The observed range of the reflection amplitudes for Seyfert galaxies ( 0.3-1.5; Zdziarski et al. 1999) is rougly consistent with the values obtained from constant pressure models $(0.6-1.8)$. Models with constant density easily produce values of the amplitude of reflection either much larger or much lower than observed.

However, such a simple model does not explain the observed correlation between the slope of the incident radiation and the amount of reflection. The observational data show a strong increase of the amount of reflection with the steepening of the incident radiation. Our set of models shows an opposite trend: a model with a steep (soft) incident radiation flux $(\alpha=1.3)$ shows a fitted amount of reflection much lower than a model with a flat (hard) incident radiation $(\alpha=0.7)$. However, our computations were done for a single value of the ionization parameter $\xi=10^{3}$ which might affect the results. We could not repeat the same computations for a practically neutral medium since for the moment our codes do not allow to solve the coupled equations of radiative transfer and energy balance if the temperature is too low.

Hydrostatic equilibrium models give lower values of the reflection component $(\sim 0.5$ for the incident spectrum slope $\alpha \sim 1$ ) than constant pressure medium, and they show a clear correlation between the slope of the incident radiation and the amount of reflection, although the range of values is somewhat smaller than observed (Done \& Nayakshin 2001).

Constant density models exhibit the biggest range in the amount of reflection (from 0 to 2), rather too large in comparison with the data.

\subsection{The Fe $K_{\alpha}$ line diagnostics}

The structure and the broadening of the iron line seen in the data is still under discussion and the situation is not clear. The broad line profile seen in the ASCA data of the source MCG-6-15-30 was confirmed in subsequent 
observations. However, the shape of the iron line in a typical Seyfert galaxy may be narrower than inferred initially (compare the results of Nandra et al. 1997 and Lubiński \& Zdziarski 2001 based on ASCA data). Recent Chandra data point towards a very narrow neutral component (e.g. Yaqoob et al. 2001a; Sambruna et al. 2001; Kaspi et al. 2001) but it may be superimposed on a broader component originating closer to the central object (Yaqoob et al. 2001b). The line is therefore most probably multicomponent but the components have different shapes and originate in different places.

Our analysis show that the iron line originating from a single region is expected to be multicomponent if the medium is at constant pressure when the slope of the incident radiation spectrum is $\alpha \sim 1$, and the medium is not neutral (i.e. the ionization parameter larger than $\sim 200$ ). The multicomponent structure originates in the same medium, so if kinematical broadening is essential, it should broaden all components similarly.

A similar multicomponent line is present in the spectra of the hydrostatic models of Różańska et al. (2002). Computations of NKK rather show a single neutral component for $\alpha \sim 1$.

A single component, either neutral or ionized, is instead characteristic for a constant density medium.

There is some level of intrinsic broadening of the line profile due to Comptonization within the reprocessing medium, as suggested by Czerny et al. (1991) but the effect is not very strong in the case of a constant pressure medium or a medium in hydrostatic equilibrium. A constant density medium broaden the line more effectively, due to the larger optical depth of the hot skin.

At present, the observations are not accurate enough to differentiate between the constant pressure and the constant density medium, if the narrow neutral component comes from the outer parts of the accretion flow (BLR and/or dusty torus) and the broad component is barely seen and not well constrained. The study of variability could help to locate the emission regions of the different components, and subsequently help to find an answer to the question of hydrostatic equilibrium.

Acknowledgements. Part of this work was supported by grant 2P03D01816 of the Polish State Committee for Scientific Research and by Jumelage/CNRS No. 16 "Astronomie France/Pologne".

\section{References}

Abrassart, A. 2000, in Proc. of the 32nd COSPAR Scientific Assembly, Nagoya, Japan, ASR, 25, 465

Abrassart, A., \& Dumont, A.-M. 1998, Proceedings of the First XMM Workshop on Science with XMM, at Noordwijk, The Netherlands, M. Dahlem (ed.), URL: http://astro.estec.esa.nl/XMM/news/ws1/ ws1_papers.html

Abrassart, A., \& Dumont, A.-M. 2000, Proceedings of the Bologna Conference on X-ray Astronomy 1999: Stellar end points, AGNs and the Diffuse X-ray Background, Astrophys. Lett. Comm., in press
Ballantyne, D. R., Ross, R. R., \& Fabian, A. C. 2001, MNRAS, 327,10

Begelman, M. C., \& McKee, C. F. 1990, ApJ, 358, 375

Begelman, M. C., McKee, C. F., \& Shields, G. A. 1983, ApJ, 271, 70

Berkley, A. J., Kazanas, D., \& Ozik, J. 2000, ApJ, 535, 712

Chiang, J., Reynolds, C. S., Blaes, O. M., et al. 2000, ApJ, 528,292

Clavel, J., Nandra, K., Makino, F., et al. 1992, ApJ, 393, 113

Collin-Souffrin, S., Czerny, B., Dumont, A.-M., \& Życki, P. T. 1996, A\&A, 314, 393

Collier, S. J., Horne, K., Kaspi, S., et al. 1998, ApJ, 500, 162

Czerny, B., \& Dumont, A.-M. 1998, A\&A, 338, 386

Czerny, B., \& Życki, P. T. 1994, ApJ, 439, L5

Czerny, B., Zbyszewska, M., \& Raine, D. J. 1991, in Iron Line Diagnostics in X-ray Sources, ed. A. Treves (SpringerVerlag, Berlin), 226

Done, C., \& Nayakshin, S. 2001, ApJ, 546, 419

Done, C., \& Życki, P. T. 1999, MNRAS, 305, 457

Done, C., Ward, M. J., Fabian, A. C., et al. 1990, MNRAS, 243,713

Dumont, A.-M., Abrassart, A., \& Collin, S. 2000, A\&A, 357, 823 (DAC)

Dumont, A.-M., \& Collin, S. 2001, in Spectroscopic Challenges of Photoionized Plasmas, ASP Conf. Ser., XXX, ed. G. Ferland, \& D. W. Savin

Edelson, R., Alexander, T., Crenshaw, D. M., et al. 1996, ApJ, 470,364

Edelson, R., Koratkar, A., Nandra, K., et al. 2000, ApJ, 534, 180

Fabian, A. C., Iwasawa, K., Reynolds, C. S., \& Young, A. J. 2000, PASP, 112, 1145

Gilfanov, M., Churazov, E., \& Revnivtsev, M. 2000, MNRAS, 316,923

Gilfanov, M., Churazov, E., \& Revnivtsev, M. 1999, A\&A, 352, 182

Green, P. 1996, ApJ, 467, 61

Hubeny, I., Blaes, O., Krolik, J. H., \& Agol, E. 2001, ApJ, 559, 680

Iwasawa, K., Fabian, A. C., Reynolds, C., et al. 1996, MNRAS, 282, 1038

Karas, V., Czerny, B., Abrassart, A., \& Abramowicz, M. A. 2000, MNRAS, 318, 547

Kaspi, S. 2001, in X-ray Emission from Accretion onto Black Holes, Proceedings of a joint workshop held by the Center for Astrophysics (Johns Hopkins University) and the Laboratory for High Energy Astrophysics (NASA/ Goddard Space Flight Center) in Baltimore, MD, June 2023, 2001, ed. T. Yaqoob, \& J. H. Krolik, published electronically (http://www.pha.jhu.edu/groups/astro/ workshop2001/)

Kazanas, D., \& Nayakshin, S. 2001, ApJ, 550, 665

Ko, Y.-K., \& Kallman, T. R. 1994, ApJ, 431, 273

Krolik, J. H., McKee, C. F., \& Tarter, C. B. 1981, ApJ, 249, 422

Laor, A., Fiore, F., Elvis, M., Wilkes, B., \& McDowell, J. C. 1997, ApJ, 477, 93

Laor, A., \& Brandt, N. W. 2002, ApJ, 569 [astro-ph/0201038]

Lee, J. C., Fabian, A. C., Brandt, W. N., Reynolds, C. S., \& Iwasawa, K. 1999, MNRAS, 310, 973

Leighly, K. M. 1999, ApJS, 125, 317

Lubiński, P., \& Zdziarski, A. A. 2001, MNRAS, 323, L37

Madej, J., \& Różańska, A. 2000, A\&A, 363, 1055 
Magdziarz, P., \& Zdziarski, A. A. 1995, MNRAS, 273, 837

Magdziarz, P., Blaes, O., Zdziarski, A. A., Johnson, W. N., \& Smith, D. A. 1998, MNRAS, 301, 179

Malzac, J. 2001, MNRAS, 325, 1625

Matt, G., Fabian, A. C., \& Ross, R. R. 1993, MNRAS, 262, 179

Mushotzky, R. F., Done, C., \& Pounds, K. A. 1993, ARA\&A, 31,717

Nandra, K., George, I. M., Mushotzky, R. F., Turner, T. J., \& Yaqoob, T. 1997, ApJ, 477, 602

Nandra, K., George, I. M., Mushotzky, R. F., Turner, T. J., \& Yaqoob, T. 1999, ApJ, 523, L17

Nandra, K., Clavel, J., Edelson, R. A., et al. 1998, ApJ, 508, 648

Nandra, K., Le, T., George, I. M., et al. 2000, ApJ, 544, 734

Nayakshin, S., Kazanas, D., \& Kallman, T. R. 2000, ApJ, 537, $833(\mathrm{NKK})$

Pounds, K. A., Nandra, K., Stewart, G. C., George, I. M., \& Fabian, A. C. 1990, Nature, 344, 132

Raymond, J. C. 1993, ApJ, 412, 267

Revnivtsev, M., Gilfanov, M., \& Churazov, E. 1999, A\&A, 347, L23

Revnivtsev, M., Gilfanov, M., \& Churazov, E. 2001, A\&A, 380, 520

Reynolds, C. S. 2000, ApJ, 533, 811

Rokaki, E., Collin-Souffrin, S., \& Magnan, C. 1993, A\&A, 272, 8

Różańska, A. 1999, MNRAS, 308, 751

Różańska, A., Czerny, B., Dumont, A.-M., \& Collin, S. 2002, MNRAS, in press

Różańska, A., \& Czerny, B. 1996, AcA, 46, 233

Ross, R. R., \& Fabian, A. C. 1993, MNRAS, 261, 74
Ruszkowski, M., Fabian, A. C., Ross, R. R., \& Iwasawa, K. 2000, MNRAS, 317, L11

Sambruna, R., Netzer, H., Kaspi, S., et al. 2001, ApJ, 546, L135

Sincell, M. W., \& Krolik, J. H. 1997, ApJ, 476, 605

Tanaka, Y., Nandra, K., Fabian, A. C., et al. 1995, Nature, 375,659

Ueda, Y., Ebisawa, K., \& Done, C. 1994, PASJ, 46, 107

Walter, R., \& Fink, H. H. 1993, A\&A, 274, 105

Wanders, I., Peterson, B. M., Alloin, D., et al. 1997, ApJS, 113,69

Weaver, K. A., Gelbord, J., \& Yaqoob, T. 2001, ApJ, 550, 261

Wilkes, B. J., Kuraszkiewicz, J., Green, P. J., Mathur, S., \& McDowell, J. C. 1999, ApJ, 513, 76

Yaqoob, T., George, I. M., Nandra, K., et al. 2001a, ApJ, 546, 759

Yaqoob, T., Padmanabhan, U., Dotani, T., et al. 2001b, in Xray Emission from Accretion onto Black Holes, Proceedings of a joint workshop held by the Center for Astrophysics (Johns Hopkins University) and the Laboratory for High Energy Astrophysics (NASA/ Goddard Space Flight Center) in Baltimore, MD, June 20-23, 2001, ed. T. Yaqoob, \& J. H. Krolik, published electronically (http://www.pha.jhu.edu/groups/astro/workshop2001/)

Yu, Q., \& Lu, Y. 2000, MNRAS, 311, 161

Zdziarski, A. A., Lubiński, P., \& Smith, D. A. 1999, MNRAS, 303, L11

Życki, P. T. 2002, MNRAS, in press [astro-ph/0202366]

Życki, P. T., \& Czerny, B. 1994, MNRAS, 266, 653

Życki, P. T., Krolik, J. H., Zdziarski, A. A., \& Kallman, T. R. 1994, ApJ, 437, 597

Życki, P. T., \& Różańska, A. 2001, MNRAS, 325, 197 